Revue d'histoire de l'Amérique française

6. REVUE D.HISTOIRE DE L'AMÉRIQUE FRANÇAISE

\title{
Papineau (Louis-Joseph) 1786-1871
}

Volume 1, numéro 1, juin 1947

URI : https://id.erudit.org/iderudit/801362ar

DOI : https://doi.org/10.7202/801362ar

Aller au sommaire du numéro

Éditeur(s)

Institut d'histoire de l'Amérique française

ISSN

0035-2357 (imprimé)

1492-1383 (numérique)

Découvrir la revue

Citer ce document

(1947). Papineau (Louis-Joseph) 1786-1871. Revue d'histoire de l'Amérique

française, 1(1), 148-151. https://doi.org/10.7202/801362ar d'utilisation que vous pouvez consulter en ligne.

https://apropos.erudit.org/fr/usagers/politique-dutilisation/ 


\title{
BIBLIOGRAPHIE 1
}

\author{
PAPINEAU (LOUIS-JOSEPH) 1786-1871
}

\section{SoURCES MANUSCRITES}

a) Archives de la Province de Québec.

Lettres de Papineau à son père et à sa mère, 1811-1852, environ 10.- Lettres de Louis-Joseph Papineau à ses frères Denis-Benjamin et Augustin, à sa sœur Rosalie, et à ses neveux, 1804-1869, environ 100.- Lettres de Louis-Joseph Papineau à ses enfants, Lactance et Amédée, 1825-1852, environ 300.- Lettres de LouisJoseph Papineau à diverses personnes, 1809-1855, environ 150.- Lettres de Joseph Papineau à sa femme, 1820-1845, environ 120 lettres.-Quantité de lettres de Denis-Benjamin Papineau à divers membres de la famille.-Grande quantité de lettres d'Amédée Papineau à ses parents et à d'autres personnages.- Journal de Lactance Papineau.- Cours de Botanique de Lactance Papineau. Notes diverses, etc.- Journal d'Amédée Papineau (dont des extraits substantiels ont été publiés dans la Presse, il y a quelques années. Une trentaine de petits volumes manuscrits renfermant des documents, lettres, etc., etc., copiés en Europe par L.-J. Papineau.Plusieurs centaines d'extraits et coupures de journaux concernant les événements de 1837-1838 et la famille Papineau.-Notes biographiques et généalogiques sur la famille Papineau. - Notes et documents sur la seigneurie de la Petite-Nation: Montebello et paroisses environnantes.-Portraits et photostats de documents divers. - Copies et originaux de lettres reçues par L.-J. et Amédée Papineau.- Quelques lettres de la famille Bourassa.-N.-B. 99\% des lettres de ce fonds sont originales, sans être toutes inédites.- (Renseignements fournis par M. Antoine Roy, archiviste de la province de Quebec).- Voir aussi: Papiers Duvernay conservés aux Archives de la Province de Québec. Rapport de l'Archiviste de la Province de Quebec, 19261927, p. 147-258.

b) Archives publiques du Canada - Ottawa.

Collection Neilson (volumes 14 et 15 en particulier) 1819-1856.-Papiers Durham (indexés).-Collection Perrault, 1835-1839, 4 volumes.-Collection Papineau-Bourassa, 1844-1866, 1 volume.- Série " $Q$ '.- Série "S".- The ElginGrey Papers 1846-1852, edited with notes and appendices by Sir Arthur G. Doughty,

1. Les “Bibliographies " qui paraîtront, autant que possible, dans chaque livraison de la Revue, ne prétendent nullement à l'exhaustion d'un sujet. Elles ne veulent être qu'une aide à la recherche. 
4 volumes, Ottawa, 1937. Consulter index à la fin du 4e volume.- Index de la Gazette de Québec.- Collection Papineau-Roebuck (indexée), 1836, 1 volume.Correspondance de Papineau, 1830-1837 (formant la partie d'un volume seulement) dans: “Upper Canada Executive Council Office Correspondence " S 3/17. - Chapman Papers: Lettres de Papineau.-(Renseignements fournis par M. Lucien Brault, directeur du service des recherches aux Archives d'Ottawa).

c) Bibliothèque de la Ville de Montréal -

Papineau (Louis-Joseph Amédée), Journal d'un fils de la liberté réfugié aux États-Unis, par suite de l'insurrection canadienne en 1837. S.I. s.éd. 1837-1843, 5 volumes. (Copie dactylographiée d'un document original). - Papineau (LouisJoseph), Lettres à John Neilson, à Robert Christie et à qưelques autres, 1824-1856 (par) Louis-Joseph Papineau. S.l. s.éd. 1824-1856. 344p.

\section{SOURCES IMFRIMEes}

a) Biographies:

Côté (Eve Circé, Mme Salomon-P.), Papineau, son influence sur la pensée canadienne: essai de psychologie historique, Montréal, Regnault, 1924, 247p. portr. David (Laurent-Olivier), Les deux Papineau, Montréal, Sénécal, 1896, 120 p.- De Celles (Alfred-Duclos), Louis-Joseph Papineau, Toronto, Morang, 1912, 203 p. portr. (The MAKERS OF CANADA).-De Celles (Alfred-Duclos), PapineauCartier, Toronto, Morang, 1906, 339 p. portr. (The MAKERS OF CANADA).De Celles (Alfred-Duclos), Papineau, 1786-1871, Montréal, Beauchemin, 1905, 243 p. portr.- Rumilly (Robert) Papineau, Monitréal, Valiquette, 1934, 281 p.Wallace (W. S.), Papineau: His place in Canadian History, published by the CANADIAN MAGAZINE, 1910!- Voir: Review of Historical Publications relating to Canada, vol. XV, Toronto, University Press, 1911, p. 67.

\section{b) Ouvrages généraux:}

Gérin-Lajoie (A.), Dix ans au Canada de 1840 à 1850, Histoire du gouvernement responsable. Québec, Demers \& Frères, 1888.- Garneau (F.-X.), Histoire du Canada.- Christie (Robert), A History of the Late province of Lower Canada parliamentary and political, from the commencement to the close of its existence as a separate province. 6 vols. Montréal, Richard Worthington, 1866.- Bibaud (M), Histoire du Canada, et des Canadiens, sous la domination anglaise, 1ère éd. I-II, Montréal, Imprimerie de Lovell et Gibson, 1844.- Chapais (Thomas), Cours d' Histoire du Canada - 1760-1867, 8 vols. Québec, Librairie Garneau, 1934.- Filteau (Gérard), Histoire des patriotes - I-II-III, Éditions Modèles, Montréal, 1942. (Documents historiques).- Kingsford (William), The History of Canada, 10 vols. Toronto, Rowsell \& Hutchison, 1898.

c) Articles ou essais:

Boucher de la Bruère (Montarville), Louis-Joseph Papineau de Saint-Denis à Paris, (dans les CAHIERS DES DIX, 1940, vol. 5, p. 79-106).- Groulx (abbé Lionel), Louis-Joseph Papineau - l'homme politiquc. (dans: Notre Maître, le Passé, 1ère série, Montréal, Granger Frères, 1941, 3e éd., p. 189-213). - Groulx (abbé Lionel), Le " Papineau » de M. Rumilly, p. 157-167; Les idées religieuses de 
Louis-Joseph Papineau, p. 167-213. (dans: Notre Mâtre, le Passé, 2e série, 2e éd., Montréal, Granger Frères, 1945).- Groulx (abbé Lionel), L'évolution de Papincau sous l'Union, (dans: Notre Maitre, le Passé, 3e série, Montréal, Granger Frères, 1944, p. 245-253). - Sulte (Benjamin), Papineau et son temps, (dans: Mélanges historiques, 1918-1932, v. 13).- Audet (Francis-J.), L'Honorable Louis-Joseph Papineau, (dans: The Canadian Historical Association, 1929, published by the Department of Public Archives, Ottawa, p. 47-56.)-Potvin (Pascal), Papineau et l'Orientation du Nationalisme québécois, (dans: The Canadian Historical Association, 1943, Edited by R. M. Saunders, Toronto, The University of Toronto Press, p. 35-42).-Potvin (Pascal). Les patriotes de 1837-1838; Essai de synthèse historique, (dans: Le Canada français, deuxième série du Parler français, publication de l'Université Laval, Québec, février, mars, avril, mai 1938). - Papineau (L.Joseph), Histoire de l'Insurrection du Canada. En réfutation du Rapport de Lord Durham. Première partie. Extraite de la Revue du Progrès, Journal publié à Paris 1839, 35p.- Sabrevois de Bleury (Charles-Clément), Refutation de l'ecrit de LouisJoseph Papineau... intitulé Histoire de l'Insurrection du Canada, publié dans le recueil hebdomadaire: La Revue du Progrès, imprimée à Paris, Montréal, John Lovell, 1839, 136p.- Read (David Breakenridge), The Canadian rebellion of 1837, 1896, voir: portrait de Louis-Joseph Papineau.-Aubin (N.), M. Louis-Joseph Papineau, (dans: Le Répertoire national ou recueil de littérature canadienne (par) J. Huston, vol. II, Montréal, Valois \& Cie,. 1893, p. 104-108).- Egerton (H. E.) and Grant (W. L.), Papineau and Mackenzie (dans: Canadian Constitutional Development, shown by selected speeches and despatches, with introductions and explanatory notes, Toronto, The Musson Book Company.- Story (Norah), Papineau in Exile, (dans: The Canadian Historical Review, new series of The Review of Historical Publications relating to Canada, Toronto, March 1929, no 1, p. 43-52).Désilets (Alphonse), "Louis-Joseph Papineau ", (dane: Le Canada français, deuxième série du Parler français, Publication de l'Université Laval, Québec, décembre 1934, p. 356-361).- Sulte (Benjamin), Papineau et son Temps, (dans: Melanges historiques, vol. 13, compilés, aninotés et publiés par Gérard Malchelosse, Montréal, G. Ducharme, 1925).- Lareau (Edmond), Louis-Joseph Papineau, (dans: Mélanges historiques et littéraires, Montréal, Eusèbe Sénécal, 1877, p. 116-129). - Bruchési (Jean), Louis-Joseph Papineau, (dans: L'Ecrin, album-souvenir de la Société SaintJean-Baptiste, édition de l'Agence Duvernay, 1945, p. 13-14).

\section{d) Periodiques:}

Journaux: Voir journaux français et anglais de l'époque de Papineau.- Voir journaux à la mort de Papineau (23 septembre 1871).- Voir (entre autres), Le Courrier du Canada, journal des intérêts canadiens, Québec, mercredi, 27 septembre 1871, lundi, 2 octobre 1871.- Voir index des journaux de l'Assemblée législative de Québec et appendices (de 1809 à 1851), au mot: Papineau.

e) Guides:

Dictionnaires biographiques: Wallace (W. Stewart), The Dictionary of Canadian Biography, Toronto, The Macmillan Company of Canada Limited, 1926. - Wallace 
(W. Stewart), The Encyclopedia of Canada, 6 vols. Toronto, University Associates of Canada, 1937.- Le Jeune (R.P. L.), Dictionnaire géneral de biographie, histoire, litterature, agriculture, commerce, industrie et des arts, sciences, mœurs, coutumes, institutions politiques et religieuses du Canada, I-II, Université d'Ottawa, 1931.Bibaud (Maximilien), Le Panthéon canadien - choix de biographies, Montréal, Jos.-M. Valois, 1891. Nouvelle éd. revue augmentée et complétée.-Bibaud (Jeune), Dictionnaire hisłorique des hommes illustres du Canada et de l'Amérique, Montréal, Bibaud et Richer, 1857. - Index: Roy (Pierre-Georges), Index du Bulletin des Recherches historiques, organe du Bureau des Archives de la Province de Québec (18951925), 4 vols. Beauceville, 1926.-Catalogue des Brochures aux Archives publiques du Canada - 1493-1877-1878-1931, avec Index. 